\title{
Women Empowerment Through Microcredit: Step Towards Alleviating Feminization of Poverty
}

\author{
Sara Wali Qazi* \\ Sindh Madressatul Islam University (SMIU) Karachi \\ Dr. Manzoor Isran* \\ SZABIST Karachi \\ Dr. Samina Isran* \\ Shah Abdul Latif University, Khairpur \\ Dr. Nadeem A. Syed \\ SZABIST Karachi
}

ABSTRACT

\begin{abstract}
Purpose - The study explores and evaluates the women's experiences with microcredit facility for the purpose of women empowerment. While there is a mutual understanding and general consensus on closer nexus between women empowerment and microcredit, women are disempowered for variety of reasons such as lacks of financial resources, being a member of patriarchy society, misinterpreted religious concepts about her role and position in the society, and cultural restraints. However, microcredit, globally promoted as a "miracle cure" for the financially weak, especially women who face so many problems accessing microcredit for variety of reason, which have been discussed in detail in this study.

Methodology/Sample - This is qualitative study with inductive approach, and interpretive philosophy which allow the existence of multiple subjective perspectives and construction of knowledge. Women who availed microcredit from microfinance institutions (MFIs) were selected for the research study. The data was congregated through in-depth interviews and stopped at saturation level. Through phenomenological analysis, women lived experiences were analyzed.

Findings - The results indicate that microcredit strengthens women in all dimensions but plays more influential role when they are provided with autonomous access to microcredit, support from family, and facilities from MFIs. Needless to say that the multidimensional women's stability contributes in alleviating feminization of poverty and this is not a social issue but the global dilemma now days.

Practical Implications - This research is significant for the microfinance practitioners to design policies for promoting women entrepreneurship and for MFIs to facilitate their contemporary women clients with trainings and efficient supervision.
\end{abstract}

Keywords: Microcredit, Women Economic Empowerment, Women Social Empowerment, Women Household increased well-being, and feminization of poverty

Jel classification: I32, I38, O12, 015

${ }^{*}$ The material presented by the authors does not necessarily portray the viewpoint of the editors and the management of the
Institute of Business \& Technology (IBT).

* Sarah Wali Qazi: swqazi@smiu.edu.pk

* Dr. Mansoor Isran: saminaisran@hotmail.com

* Dr. Samina Isran: saminaisran@ hotmail.com

* Dr. Nadeem A. Syed, nadeem@szabist.edu.pk

C JMSS is published by the Institute of Business and Technology (IBT).

Main Ibrahim Hydri Road, Korangi Creek, Karachi-75190, Pakistan. 


\section{INTRODUCTION}

Women empowerment is the influential subject matter in the current era. Before starting discussing the women empowerment, it is necessary to understand how women are disempowered. Disempowering is a process which reduces the power or authority of an individual or group in making crucial decisions and shapes their lives accordingly. According to the philosophers, researchers and professors in the particular field of gender studies, disempowerment is not only the issue associated with the developing countries but the developed countries women also facing hurdles in gaining power. There are so many reasons which come in any women way is lack of education, religion interpreters, patriarchal societies, lack of employment opportunities, lack of financial resources etc.Most of the religious interpreters misguide the religious beliefs and suppressed, ignored and abused the women. Religion guidance are taken into negative way that women are believed to be inferior to men and considered the second-class category person. (Sinclair, 2012)(Bhat \& Lakshmi, 2002)

National Patriarchal society is the place where women are not only under the men's power but are also subject to discrimination, dishonor, exploitations, control, subjugation and violence. Women are even deprived of equal treatment in terms of basic necessities like right to food, education, employment, healthcare, and decision making power (Ray, 1994.) Women in patriarchal society face more hurdles in developing and sustaining their own identity and due to these factors; skilled and capable women are forcibly excluded from economic activities.(Sinclair, 2012). And whatever labour they perform is not recognized, nor accounted for in the statistical figures.

In Pakistan, the role of women is still under scrutiny in spite of $21^{\text {st }}$ century dawning. While in most of the emerging economies role of women is increasingly being redefined, women in Pakistan sadly are still faced with cultural and religious taboos despite the fact they comprise almost half of the population and contributing strongly to the national economy though mostly informally. Despite being significant contributors their status is vulnerable due to the few factors like their mobility, education, lack of financial funds, others like social restraints and minimal institutional support and many more, these may lead them to disempowerment (Sinclair, 2012).

However, with passage of time despite such depressing state of affairs for the women in this country, they are subject to a demand of more financial contribution towards their families. Accordingly the provision of sources like microfinance is being seen as relevant. This paper discusses the impact of microcredit on women empowerment which will be measured through the qualitative indicators as not a single quantitative technique has a capacity to articulate these indicators like power to decide, self-direction or autonomy. The unavailability of credit is the main barrier in developing women as the powerful asset of the nation. Microfinance is designed for the people who belong to the weak social and economic community, and who are unable to meet their financial needs. Although this concept was initiated in 1700 with the basic theme was to provide the financial assistance to the poor people (CGAP, 2006) on the pattern of Grameen Bank in Bangladesh.

Accordingly, this research work is based on following two assumptions:

a. Women attain economic empowerment with their access to microcredit. Family support and appropriate monitoring and trainings empower women in a social context but they also experience progress in their overall household well-being. These three perspectives - economic, social and improvement in living standards - collectively empower women in all dimensions.

b. Empowerment of women signifies the step towards alleviating feminization of poverty 


\section{LITERATURE REVIEW}

Empowerment is concerned with the development of human and the way they face challenges and involved themselves in the establishment process for reducing the work burden (Luttrell, Quiroz, Scrutton, \& Bird, 2009). Empowerment is also concerned with the availability of choice, and power of choosing among the alternatives (Mayoux, 2005). Women empowerment is the growth of freedom of preference and achievement to shape one's life (Nasir, 2013).

Women empowerment has multiple dimensions. The first dimension is their economic stability - they are competent to participate in the economy and make better decisions for their economic position (Mayoux, 2005; Gola et al, 2011). The second dimension of women empowerment is their ability to prevent the men from being spendthrift. Living standard can be improved if both men and women contribute momentous income and share responsibilities. Welfare interventions provide financial services and also emphasize on women health, security, and education which augment their abilities and skills. The third dimension in this regard is social empowerment in which women have power to take decision, remaining autonomous, self-confident and manage their household independently. Through these determinants they can interact within the society and specified quota seats in assembly for women, empower them politically (Lemire, Pearson, \& Campbell, 2001)(Mayoux, 2005).

\subsection{Microfinance and Women Empowerment}

The microfinance paradigms have their own aims and interpretation, with main concern of delivering credit with corresponding services and providing appropriate structure to small organizations. Regarding women empowerment, central aim of this paradigm has been providing the poor women with credit facilities. The main instruments of this model are small credits provisions to underprivileged people including women. It encourages female participation, hypothesizes women sustainability, decreases in poverty and social enhancement are naturally collaborative (Mayoux, 2006).

In the earlier history of micro-financing, Jonathan Swift, in early 1700, along with other nationalist of Ireland initiated the Microfinance Institutions (MFIs) which provided financial support to poor of rural areas era. Spooner, in mid-1800, highlighted microcredit, as best way of poverty alleviation. In 1970 s, microcredit programs emerged in developing countries with the purpose of providing loans to deprived women to enable them to invest in small enterprises. In 1976, Professor Muhammad Younus in Chittagong University designed an experimental credit program to serve the poor. In 1983 he launched Grameen Bank, which presently has more than 4 million clients.

In 1980, Aga Khan Rural Support Program initiated. National Rural Support Program and Sarhad Rural Support Program were established in the microfinance sector. These Rural Support Programs were introduced to provide financial services to poor. Pakistan Microfinance Network (PMN) originated in 1995 and began to play a role as an emergent Microfinance Provider (MFP). In 2000, Pakistan Poverty Alleviation Fund (PPAF) provided its first loan to MFI, and State Bank of Pakistan (SBP) initiated a microfinance unit. Thirteen MFBs got licenses by 2012 and different NGO's were also established however researches reveal demand of such MFIs is far greater than available still. Presently, Taraqee Foundation's (TFs) Micro-Finance and Economic Development program (MFED) is doing a good job in Pakistan whose main motto is to alleviate poverty through microcredit and saving facilities to both women and men to support gender equality. This foundation used Road Journeys model that showed that women take initiative in setting their enterprises though they have faced certain challenges including family opposition and demands.

In 2010, the then Pakistani President signed the bill namely 'Protection against Harassment of Women at Workplace Bill 2009', which was highly praised by the human rights commission of Pakistan and adopted by parliament on January, 2010. On 2012, two more bills were signed that prevent women to be used as tradable commodity. In Pakistan, the change was needed not only from the legislative corridors but also from the civil society organizations that they in cooperation with each other must eliminate 
these social biases, social dilemmas, empower women and represent them as the significant part of country (Hali, 2013). Besides, government of Pakistan also initiated the "Benazir Income Support Programme", the direct disbursement of support to the poor women and through it they enjoy better nutrient and participate courageously in development of the nation (BISP: At a Glance, 2007). However, despite these and other initiative by the successive governments, the issue of minimum access to financial resources for the women always persisted.

\subsection{Theoretical Framework}

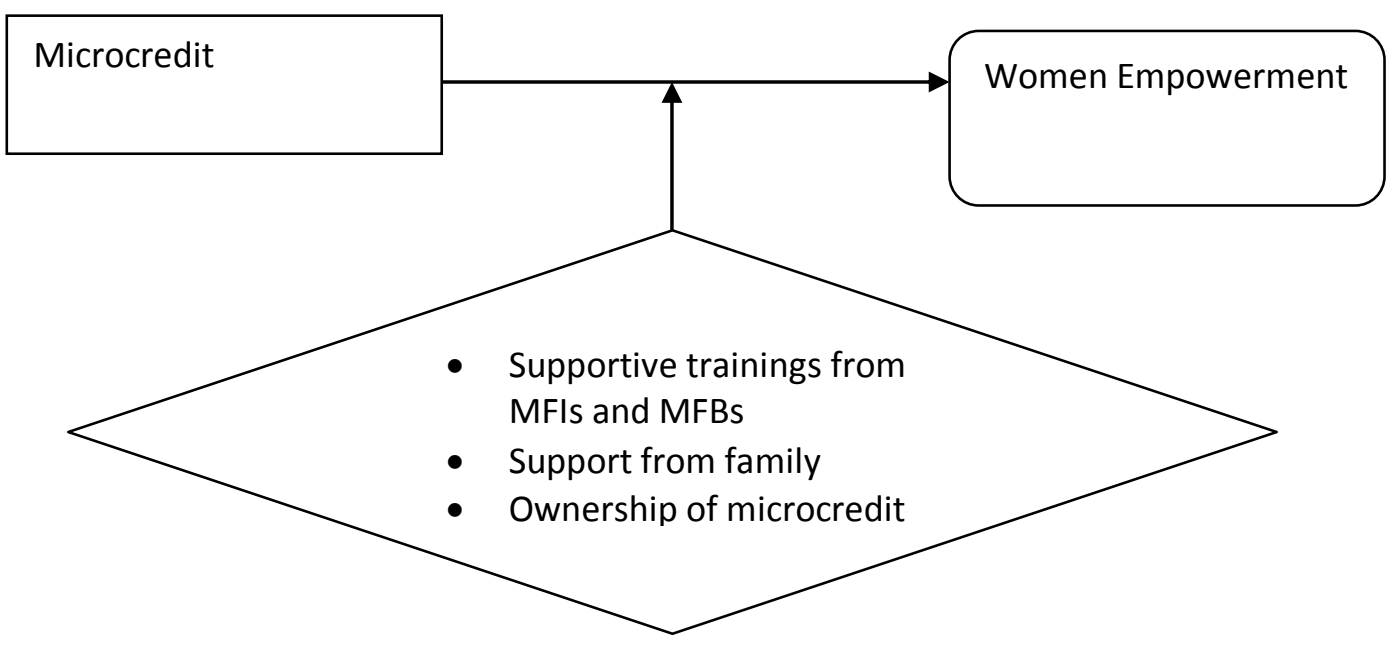

Theoretical framework shows the link between micro credit (independent variable) and women empowerment (dependent variable). External support in various forms like ownership of microcredit to women, family support and MFIs financial literacy trainings, vocational skill developments trainings etc. is the mediating variable that may have augmenting or moderating effect and influences the strength of the relationship between the variables.

\section{RESEARCH METHODOLOGY}

This research work is qualitative in nature with phenomenological research design that investigated the respondents' live experiences with microcredit program. Interpretivism philosophy with inductive approach was adopted in this work (Saunders et al, 2009).

Seventeen women from the underprivileged areas of Karachi like Mehmoodabad, Korangi, Malir, Saudabad, and Orangi who had availed microcredit purposively for respondents for this research work. They were selected through non-probability sampling technique in its purposive mode. Out of these seventeen women, thirteen women were running their own businesses and two of them were doing jobs and had availed credit for their male family members. Eleven of the participants had efficiently implemented their business and contributed significant amount to the household. They also had future plans for expanding their businesses. Their businesses had generated employment opportunities for other women. For instance, one of them had opened a school wherein 6 female teachers from the same locality had been employed. Four of the respondents had had microfinance loan but transferred it to their male family members, hence did not yield any economic empowerment out of it.

These women were interviewed in-depth to know about their experience with microcredit programs and data was integrated in the form of matrix. Phenomenological analysis was applied for transcribing and analyzing the data. 


\section{DATA INTEGRATION}

\subsection{Women Economic Empowerment Indicators}

\begin{tabular}{|c|c|}
\hline Elements & Indicators \\
\hline \multirow[t]{3}{*}{$\begin{array}{l}\text { Women's } \\
\text { Participation in } \\
\text { economy }\end{array}$} & $\begin{array}{l}\text { Establishing } \\
\text { business } \\
\text { (increased } \\
\text { women } \\
\text { entrepreneurs) }\end{array}$ \\
\hline & $\begin{array}{lr}\text { Ownership } & \text { of } \\
\text { Credit } & \text { for } \\
\text { economic } & \\
\text { activities } & \end{array}$ \\
\hline & $\begin{array}{l}\text { Creating work } \\
\text { opportunities for } \\
\text { other women's }\end{array}$ \\
\hline \multirow[t]{3}{*}{$\begin{array}{l}\text { Women's } \\
\text { Success }\end{array}$} & $\begin{array}{l}\text { Effectively and } \\
\text { efficiently } \\
\text { implemented the } \\
\text { business } \\
\text { practices }\end{array}$ \\
\hline & $\begin{array}{l}\text { Support from } \\
\text { family members } \\
\text { for economic } \\
\text { activities }\end{array}$ \\
\hline & $\begin{array}{l}\text { Growth in } \\
\text { business }\end{array}$ \\
\hline \multirow[t]{3}{*}{$\begin{array}{ll}\text { Control over } \\
\text { assets }\end{array}$} & $\begin{array}{l}\text { Ownership of } \\
\text { Productive assets }\end{array}$ \\
\hline & $\begin{array}{l}\text { Contribution to } \\
\text { household } \\
\text { income }\end{array}$ \\
\hline & $\begin{array}{lr}\text { Control over } \\
\text { some cash and } \\
\text { savings }\end{array}$ \\
\hline \multirow{3}{*}{$\begin{array}{l}\text { Productivity and } \\
\text { skills }\end{array}$} & Access to Market \\
\hline & $\begin{array}{l}\text { Access } \\
\text { vocational } \\
\text { trainings } \\
\end{array}$ \\
\hline & $\begin{array}{lr}\text { Access } & \text { to } \\
\text { financial literacy } \\
\text { trainings }\end{array}$ \\
\hline
\end{tabular}

\subsection{Women Social Empowerment Indicators}

Women social empowerment can be measured through these indicators (Mayoux, 2005)(Nair, 2013):

\begin{tabular}{|l|l|}
\hline Elements & Indicators \\
\hline & \\
\hline Decision Making & Women \\
& $\begin{array}{l}\text { involvement in } \\
\text { households } \\
\text { decision making }\end{array}$ \\
\hline
\end{tabular}




\begin{tabular}{|c|c|}
\hline & $\begin{array}{l}\text { Women } \\
\text { involvement in } \\
\text { making decisions } \\
\text { about their family } \\
\text { size }\end{array}$ \\
\hline & $\begin{array}{l}\text { Taking decisions } \\
\text { for themselves }\end{array}$ \\
\hline \multirow[t]{2}{*}{$\begin{array}{l}\text { Self-sufficiency } \\
\& \text { Mobility }\end{array}$} & $\begin{array}{ll}\begin{array}{l}\text { Freedom } \\
\text { movement }\end{array} & \text { to } \\
\end{array}$ \\
\hline & Autonomous \\
\hline \multirow{3}{*}{$\begin{array}{l}\text { Status within the } \\
\text { community and } \\
\text { at home/ Respect }\end{array}$} & $\begin{array}{l}\text { Respect in the } \\
\text { family }\end{array}$ \\
\hline & $\begin{array}{l}\text { Respect in the } \\
\text { neighborhood }\end{array}$ \\
\hline & $\begin{array}{l}\text { Encouragement } \\
\text { factor for other } \\
\text { women in the } \\
\text { society }\end{array}$ \\
\hline Self-confidence & $\begin{array}{l}\text { Ability to take } \\
\text { challenges and } \\
\text { believe in own } \\
\text { self }\end{array}$ \\
\hline
\end{tabular}

\subsection{Women Improved Social Living Indicators}

The list of indicators that measure the enhanced living standards (Golla, Malhotra, Nanda, \& Mehra, 2011)(Mayoux, 2005):

\begin{tabular}{|c|c|}
\hline $\begin{array}{l}\text { Qualitative } \\
\text { variables }\end{array}$ & Indicators \\
\hline $\begin{array}{l}\text { Men } \\
\text { contribution } \\
\text { of income }\end{array}$ & $\begin{array}{l}\text { Female } \\
\text { households }\end{array}$ \\
\hline \multirow[t]{3}{*}{$\begin{array}{l}\text { Increased } \\
\text { Children } \\
\text { Well-being }\end{array}$} & Improved Nutrition \\
\hline & $\begin{array}{l}\text { Access to better health } \\
\text { facilities }\end{array}$ \\
\hline & $\begin{array}{l}\text { Access to better } \\
\text { education }\end{array}$ \\
\hline \multirow{2}{*}{$\begin{array}{l}\text { Increased } \\
\text { Women } \\
\text { Own well- } \\
\text { being }\end{array}$} & $\begin{array}{l}\text { Proportion of income } \\
\text { spent on themselves }\end{array}$ \\
\hline & $\begin{array}{l}\text { Improved nutrition, } \\
\text { and health }\end{array}$ \\
\hline \multirow{2}{*}{$\begin{array}{l}\text { Increased } \\
\text { Men well- } \\
\text { being }\end{array}$} & Happiness at home \\
\hline & $\begin{array}{l}\text { Reduce pressure of } \\
\text { earnings }\end{array}$ \\
\hline
\end{tabular}


Sara Wali Qazi, Manzoor Isran, Samina Isran, Nadeem A. Syed

\begin{tabular}{|l|l|}
\hline & $\begin{array}{l}\text { Women increased } \\
\text { control on decision } \\
\text { making prevent men to } \\
\text { spend in unproductive } \\
\text { and harmful. }\end{array}$ \\
\hline $\begin{array}{l}\text { Poverty } \\
\text { reduction }\end{array}$ & $\begin{array}{l}\text { Improved Overall } \\
\text { household wellbeing }\end{array}$ \\
\hline & Decrease vulnerability \\
\hline
\end{tabular}




\section{PHENOMENOLOGICAL ANALYSIS}

\begin{tabular}{|c|c|c|c|c|c|c|}
\hline Categories & Case \# 1 & Case \# 2 & Case \# 3 & Case \# 4 & Case \# 5 & Case \# 6 \\
\hline $\begin{array}{l}\text { Women's } \\
\text { Participation } \\
\text { in economy }\end{array}$ & $\begin{array}{l}\text { Participatin } \\
\text { g by running } \\
\text { school }\end{array}$ & $\begin{array}{l}\text { Stitching T- } \\
\text { shirts }\end{array}$ & $\begin{array}{l}\text { Running } \\
\text { beauty } \\
\text { parlor }\end{array}$ & $\begin{array}{l}\text { Running } \\
\text { two } \\
\text { garments } \\
\text { shop }\end{array}$ & $\begin{array}{l}\text { Running } \\
\text { undergarme } \\
\text { nts shop }\end{array}$ & $\begin{array}{l}\text { Running } \\
\text { uniforms } \\
\text { shop }\end{array}$ \\
\hline $\begin{array}{l}\text { Women's } \\
\text { Success }\end{array}$ & $\begin{array}{l}\text { Yes, } \\
\text { successful }\end{array}$ & $\begin{array}{l}\text { Yes, } \\
\text { successful }\end{array}$ & $\begin{array}{l}\text { Yes, } \\
\text { successful }\end{array}$ & $\begin{array}{l}\text { Yes, } \\
\text { successful }\end{array}$ & $\begin{array}{l}\text { Yes, } \\
\text { successful }\end{array}$ & $\begin{array}{l}\text { Yes, } \\
\text { successful }\end{array}$ \\
\hline $\begin{array}{l}\text { Control over } \\
\text { assets }\end{array}$ & $\begin{array}{l}\text { Yes, } \\
\text { investing, } \\
\text { owing assets } \\
\text { and saving }\end{array}$ & $\begin{array}{l}\text { Yes, } \\
\text { investing, } \\
\text { owing assets } \\
\text { \& saving }\end{array}$ & $\begin{array}{l}\text { Yes, } \\
\text { investing, } \\
\text { owing assets } \\
\text { \& saving }\end{array}$ & $\begin{array}{l}\text { Yes, } \\
\text { investing, } \\
\text { owing assets } \\
\text { \& saving }\end{array}$ & $\begin{array}{l}\text { Yes, saving } \\
\text { for investing }\end{array}$ & $\begin{array}{l}\text { No savings, } \\
\text { no assets }\end{array}$ \\
\hline $\begin{array}{l}\text { Productivity } \\
\text { and skills }\end{array}$ & $\begin{array}{l}\text { No trainings } \\
\text { from MFIs, } \\
\text { but self- } \\
\text { learner }\end{array}$ & $\begin{array}{l}\text { No } \\
\text { trainings } \\
\text { from MFIs }\end{array}$ & $\begin{array}{l}\text { No trainings } \\
\text { from MFIs }\end{array}$ & $\begin{array}{l}\text { Financial } \\
\text { literacy and } \\
\text { skill } \\
\text { developmen } \\
\text { t trainings }\end{array}$ & $\begin{array}{l}\text { Financial } \\
\text { Literacy } \\
\text { trainings }\end{array}$ & $\begin{array}{l}\text { No } \\
\text { vocational } \\
\text { trainings, } \\
\text { only } \\
\text { financial } \\
\text { literacy } \\
\text { trainings }\end{array}$ \\
\hline \multicolumn{7}{|c|}{ Women Economic Empowerment } \\
\hline $\begin{array}{l}\text { Decision } \\
\text { Making } \\
\text { Power }\end{array}$ & $\begin{array}{l}\text { Yes, took } \\
\text { family } \\
\text { decisions }\end{array}$ & $\begin{array}{l}\text { Husband } \\
\text { and wife } \\
\text { took } \\
\text { decisions }\end{array}$ & $\begin{array}{l}\text { Yes, took } \\
\text { decisions for } \\
\text { husband too }\end{array}$ & $\begin{array}{l}\text { Yes, took } \\
\text { family } \\
\text { decisions } \\
\text { and } \\
\text { business }\end{array}$ & $\begin{array}{l}\text { No, her son } \\
\text { took } \\
\text { decisions }\end{array}$ & $\begin{array}{l}\text { Husband } \\
\text { and wife } \\
\text { took } \\
\text { decisions }\end{array}$ \\
\hline $\begin{array}{l}\text { Self- } \\
\text { sufficiency } \\
\& \text { Mobility }\end{array}$ & $\begin{array}{l}\text { Sociable } \\
\text { and } \\
\text { Independen } \\
\mathrm{t}\end{array}$ & $\begin{array}{l}\text { Not much } \\
\text { sociable but } \\
\text { encourage } \\
\text { others }\end{array}$ & $\begin{array}{l}\text { Sociable } \\
\text { and } \\
\text { Independen } \\
\mathrm{t}\end{array}$ & $\begin{array}{l}\text { Sociable } \\
\text { and } \\
\text { Independen } \\
\mathrm{t}\end{array}$ & $\begin{array}{l}\text { Sociable } \\
\text { and } \\
\text { Independen } \\
t\end{array}$ & $\begin{array}{l}\text { Sociable } \\
\text { and active }\end{array}$ \\
\hline $\begin{array}{l}\text { Status } \\
\text { within the } \\
\text { community } \\
\text { and at } \\
\text { home/ } \\
\text { Resnect }\end{array}$ & $\begin{array}{l}\text { Recognized } \\
\text { and } \\
\text { respectable }\end{array}$ & $\begin{array}{l}\text { Recognized } \\
\text { and } \\
\text { respectable }\end{array}$ & $\begin{array}{l}\text { Recognized } \\
\text { and } \\
\text { respectable }\end{array}$ & $\begin{array}{l}\text { Recognized, } \\
\text { respectable } \\
\& \text { most } \\
\text { influencing } \\
\text { character }\end{array}$ & $\begin{array}{l}\text { Recognized } \\
\text { and } \\
\text { respectable }\end{array}$ & $\begin{array}{l}\text { Enhanced } \\
\text { value and } \\
\text { respect at } \\
\text { home }\end{array}$ \\
\hline $\begin{array}{l}\text { Self- } \\
\text { confidence }\end{array}$ & Yes & no & yes & Yes & yes & Yes \\
\hline \multicolumn{7}{|c|}{ Women Social empowerment } \\
\hline $\begin{array}{l}\text { Men } \\
\text { contribution } \\
\text { of income }\end{array}$ & $\begin{array}{l}\text { Single, and } \\
\text { Brother } \\
\text { contribute }\end{array}$ & $\begin{array}{l}\text { Husband } \\
\text { contribute } \\
\text { equally }\end{array}$ & $\begin{array}{l}\text { Husband } \\
\text { contribute } \\
\text { less than her }\end{array}$ & $\begin{array}{l}\text { Men } \\
\text { withdraw } \\
\text { his } \\
\text { contribution }\end{array}$ & $\begin{array}{l}\text { Yes, her son } \\
\text { contributes }\end{array}$ & $\begin{array}{l}\text { Husband } \\
\text { contribute }\end{array}$ \\
\hline
\end{tabular}




\begin{tabular}{|c|c|c|c|c|c|c|}
\hline $\begin{array}{l}\text { Increased } \\
\text { Children } \\
\text { Well-being }\end{array}$ & $\begin{array}{l}\text { Improved } \\
\text { Siblings } \\
\text { wellbeing }\end{array}$ & yes & yes & Yes & Yes & Yes \\
\hline $\begin{array}{l}\text { Increased } \\
\text { Women } \\
\text { Own well- } \\
\text { being }\end{array}$ & $\begin{array}{l}\text { Yes, she } \\
\text { had done } \\
\text { masters } \\
\text { after loan }\end{array}$ & yes & Yes & Yes & Yes & Yes \\
\hline $\begin{array}{l}\text { Increased } \\
\text { Men well- } \\
\text { being }\end{array}$ & $\begin{array}{l}\text { Sharing } \\
\text { brother } \\
\text { responsibilit }\end{array}$ & yes & yes & No & Widowed & Yes \\
\hline $\begin{array}{l}\text { Poverty } \\
\text { reduction }\end{array}$ & Yes, & yes & yes & Yes & Yes & Yes \\
\hline \multicolumn{7}{|c|}{ Enhanced Living Standard } \\
\hline Categories & Case \# 7 & Case \# 8 & Case \# 9 & Case \# 10 & Case \# 11 & Case \# 12 \\
\hline $\begin{array}{l}\text { Women's } \\
\text { Participation } \\
\text { in economy }\end{array}$ & $\begin{array}{l}\text { Loan } \\
\text { transfer } \\
\text { running } \\
\text { husband's } \\
\text { business, } \\
\text { supporting } \\
\text { husband }\end{array}$ & $\begin{array}{l}\text { Loan } \\
\text { transfer } \\
\text { running } \\
\text { husband's } \\
\text { business }\end{array}$ & $\begin{array}{l}\text { Loan } \\
\text { transfer to } \\
\text { father-in- } \\
\text { law for } \\
\text { burger cart }\end{array}$ & $\begin{array}{l}\text { Running her } \\
\text { artificial } \\
\text { flower and } \\
\text { vase } \\
\text { business }\end{array}$ & $\begin{array}{l}\text { Paper bags } \\
\text { business }\end{array}$ & $\begin{array}{l}\text { Running a } \\
\text { work } \\
\text { business }\end{array}$ \\
\hline $\begin{array}{l}\text { Women's } \\
\text { Success }\end{array}$ & $\begin{array}{l}\text { Not her } \\
\text { own growth }\end{array}$ & $\begin{array}{l}\text { Not her } \\
\text { own growth }\end{array}$ & No & $\begin{array}{l}\text { Yes, } \\
\text { magnificent } \\
\text { success }\end{array}$ & No & Yes \\
\hline $\begin{array}{l}\text { Control over } \\
\text { assets }\end{array}$ & No & No & No & Yes & No & Yes \\
\hline $\begin{array}{l}\text { Productivity } \\
\text { and skills }\end{array}$ & $\begin{array}{l}\text { Expense } \\
\text { and budget } \\
\text { trainings }\end{array}$ & No & No & $\begin{array}{l}\text { No trainings } \\
\text { from MFB }\end{array}$ & No & Yes \\
\hline \multicolumn{7}{|c|}{ Women Economic Empowerment } \\
\hline $\begin{array}{l}\text { Decision } \\
\text { Making } \\
\text { Power }\end{array}$ & $\begin{array}{l}\text { Husband } \\
\text { took } \\
\text { decisions }\end{array}$ & $\begin{array}{l}\text { Husband } \\
\text { took } \\
\text { decisions }\end{array}$ & $\begin{array}{l}\text { Father-in- } \\
\text { law took } \\
\text { decisions }\end{array}$ & $\begin{array}{l}\text { Widowed, } \\
\text { Took all } \\
\text { decision }\end{array}$ & $\begin{array}{l}\text { Husband } \\
\text { took } \\
\text { decisions }\end{array}$ & $\begin{array}{l}\text { Her mother } \\
\text { took } \\
\text { decisions for } \\
\text { her (single } \\
\text { girl) }\end{array}$ \\
\hline $\begin{array}{l}\text { Self- } \\
\text { sufficiency } \\
\& \text { Mobility }\end{array}$ & $\begin{array}{l}\text { Reserve, } \\
\text { dependent } \\
\text { on husband }\end{array}$ & $\begin{array}{l}\text { Reserve, } \\
\text { dependent } \\
\text { on husband }\end{array}$ & Reserve & $\begin{array}{l}\text { Sociable, } \\
\text { active and } \\
\text { self-directed }\end{array}$ & $\begin{array}{l}\text { Reserve, } \\
\text { dependent } \\
\text { on husband }\end{array}$ & $\begin{array}{l}\text { Not much } \\
\text { sociable but } \\
\text { encourage } \\
\text { others }\end{array}$ \\
\hline
\end{tabular}




\begin{tabular}{|c|c|c|c|c|c|c|c|c|}
\hline \multirow{2}{*}{$\begin{array}{l}\text { Status } \\
\text { within the } \\
\text { community } \\
\text { and at } \\
\text { home/ } \\
\text { Resnect } \\
\text { Self- } \\
\text { confidence }\end{array}$} & $\begin{array}{l}\text { Respect at } \\
\text { home }\end{array}$ & $\begin{array}{l}\text { Respect at } \\
\text { home }\end{array}$ & \multicolumn{2}{|c|}{$\begin{array}{l}\text { No change } \\
\text { after loan }\end{array}$} & \multicolumn{2}{|c|}{$\begin{array}{l}\text { Recognized, } \\
\text { respectable } \\
\text { and most } \\
\text { influencing } \\
\text { character in } \\
\text { the }\end{array}$} & $\begin{array}{l}\text { No change } \\
\text { after loan }\end{array}$ & \multirow[t]{2}{*}{$\begin{array}{l}\text { Respectable } \\
\text { and } \\
\text { influencing } \\
\text { character in } \\
\text { the } \\
\text { neighborho } \\
\text { Yes }\end{array}$} \\
\hline & No & No & \multicolumn{2}{|l|}{ No } & \multicolumn{2}{|c|}{ Yes } & Yes & \\
\hline \multicolumn{9}{|c|}{ Women Social empowerment } \\
\hline $\begin{array}{l}\text { Men } \\
\text { contribution } \\
\text { of income }\end{array}$ & \begin{tabular}{|l}
$100 \%$ \\
earnings \\
from \\
husband
\end{tabular} & $\begin{array}{l}\text { Only from } \\
\text { husband }\end{array}$ & \multicolumn{2}{|c|}{$\begin{array}{l}\text { Husband } \\
\text { gave his } \\
\text { salary } \\
\text { father-in- } \\
\text { law now not } \\
\text { doing any } \\
\text { business }\end{array}$} & \multicolumn{2}{|l|}{ 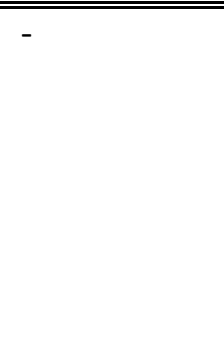 } & Yes & $\begin{array}{l}\text { Yes, father } \\
\text { is doing job } \\
\text { but not so } \\
\text { much } \\
\text { contribution }\end{array}$ \\
\hline $\begin{array}{l}\text { Increased } \\
\text { Children } \\
\text { Well-being }\end{array}$ & $\begin{array}{l}\text { Only } \\
\text { nutrition } \\
\text { and health } \\
\text { facilities, no } \\
\text { education } \\
\end{array}$ & No & \multicolumn{2}{|l|}{ No } & \multicolumn{2}{|l|}{ Yes } & No & $\begin{array}{l}\text { Improved } \\
\text { Siblings } \\
\text { wellbeing }\end{array}$ \\
\hline $\begin{array}{l}\text { Increased } \\
\text { Women } \\
\text { Own well- } \\
\text { being }\end{array}$ & No & No & \multicolumn{2}{|l|}{ No } & \multicolumn{2}{|l|}{ Yes } & No & Yes \\
\hline $\begin{array}{l}\text { Increased } \\
\text { Men well- } \\
\text { being }\end{array}$ & Yes & No & \multicolumn{2}{|l|}{ No } & \multicolumn{2}{|l|}{-} & No & Yes \\
\hline $\begin{array}{l}\text { Poverty } \\
\text { reduction }\end{array}$ & $\begin{array}{l}\text { Yes but } \\
\text { little bit }\end{array}$ & no & \multicolumn{2}{|l|}{ No } & \multicolumn{2}{|c|}{$\begin{array}{l}\text { Yes, terrific } \\
\text { reduction }\end{array}$} & No & Yes \\
\hline \multicolumn{9}{|c|}{ Enhance Living Standard } \\
\hline Categories & Case \# 13 & \multicolumn{2}{|c|}{ Case \# 14} & \multicolumn{2}{|c|}{ Case \# 15} & \multicolumn{2}{|c|}{ Case \# 16} & Case \# 17 \\
\hline $\begin{array}{l}\text { Women's } \\
\text { Participation in } \\
\text { economy }\end{array}$ & $\begin{array}{l}\text { First credit } \\
\text { transfer to } \\
\text { husband \& } 2^{\text {nd }} \\
\text { loan for } \\
\text { running her } \\
\text { embroidery } \\
\text { business }\end{array}$ & \multicolumn{2}{|c|}{$\begin{array}{l}\text { Transfer credit } \\
\text { to her son for } \\
\text { running } \\
\text { business, } \\
\text { working } \\
\text { woman } \\
\text { (widow) }\end{array}$} & $\begin{array}{l}\text { Runn } \\
\text { embr } \\
\text { busin } \\
\text { with j }\end{array}$ & $\begin{array}{l}\text { ng her } \\
\text { idery } \\
\text { ss along } \\
\text { b }\end{array}$ & & $\begin{array}{l}\text { ining her } \\
\text { proidery } \\
\text { iness }\end{array}$ & $\begin{array}{l}\text { Transfer loan } \\
\text { to husband } \\
\text { and husband } \\
\text { now sit idle }\end{array}$ \\
\hline $\begin{array}{l}\text { Women's } \\
\text { Success }\end{array}$ & Yes & No & & Yes & & Yes & & No \\
\hline
\end{tabular}




\begin{tabular}{|c|c|c|c|c|c|}
\hline $\begin{array}{l}\text { Control over } \\
\text { assets }\end{array}$ & Yes & Yes & Yes & Yes & No \\
\hline $\begin{array}{l}\text { Productivity } \\
\text { and skills }\end{array}$ & No & No & $\begin{array}{l}\text { Yes, easily } \\
\text { access to } \\
\text { market and } \\
\text { havo }\end{array}$ & $\begin{array}{l}\text { Yes, easy } \\
\text { access to } \\
\text { market }\end{array}$ & No \\
\hline \multicolumn{6}{|c|}{ Women Economic Empowerment } \\
\hline $\begin{array}{l}\text { Decision } \\
\text { Making Power }\end{array}$ & $\begin{array}{l}\text { Yes took all } \\
\text { decisions }\end{array}$ & $\begin{array}{l}\text { Yes took all } \\
\text { decisions }\end{array}$ & $\begin{array}{l}\text { She took all } \\
\text { decisions }\end{array}$ & $\begin{array}{l}\text { Both husband } \\
\text { wife took } \\
\text { decisions }\end{array}$ & $\begin{array}{l}\text { Husband took } \\
\text { decisions }\end{array}$ \\
\hline $\begin{array}{l}\text { Self-sufficiency } \\
\text { \& Mobility }\end{array}$ & $\begin{array}{l}\text { Sociable \& } \\
\text { active }\end{array}$ & $\begin{array}{l}\text { Sociable \& } \\
\text { active }\end{array}$ & $\begin{array}{l}\text { Sociable, } \\
\text { active and Self- } \\
\text { directed }\end{array}$ & $\begin{array}{l}\text { Sociable \& } \\
\text { active }\end{array}$ & $\begin{array}{l}\text { Reserve, } \\
\text { inactive and sit } \\
\text { at home }\end{array}$ \\
\hline $\begin{array}{l}\text { Status within } \\
\text { the community } \\
\text { and at home/ } \\
\text { Respect }\end{array}$ & $\begin{array}{l}\text { Recognized } \\
\text { and } \\
\text { respectable }\end{array}$ & $\begin{array}{l}\text { Recognized } \\
\text { and } \\
\text { respectable } \\
\text { due to her job } \\
\text { in clinic }\end{array}$ & $\begin{array}{l}\text { Recognized } \\
\text { and } \\
\text { respectable }\end{array}$ & $\begin{array}{l}\text { Recognized } \\
\text { and } \\
\text { respectable }\end{array}$ & $\begin{array}{l}\text { No enhanced } \\
\text { respect at } \\
\text { home and } \\
\text { society }\end{array}$ \\
\hline $\begin{array}{l}\text { Self- } \\
\text { confidence }\end{array}$ & Yes & Yes & Yes & Yes & No \\
\hline \multicolumn{6}{|c|}{ Women Social empowerment } \\
\hline $\begin{array}{l}\text { Men } \\
\text { contribution of } \\
\text { income }\end{array}$ & $\begin{array}{l}\text { Yes, equal } \\
\text { contribution }\end{array}$ & $\begin{array}{l}\text { Yes, son } \\
\text { contribute } \\
\text { more than her }\end{array}$ & $\begin{array}{l}\text { No, her } \\
\text { husband is not } \\
\text { living with her }\end{array}$ & $\begin{array}{l}\text { Yes, her } \\
\text { contribution is } \\
\text { more than her } \\
\text { husband }\end{array}$ & $\begin{array}{l}\text { Only man } \\
\text { earns }\end{array}$ \\
\hline $\begin{array}{l}\text { Increased } \\
\text { Children Well- } \\
\text { being }\end{array}$ & Yes & Yes & $\begin{array}{l}\text { No children, } \\
\text { living with } \\
\text { parents }\end{array}$ & Yes & No \\
\hline $\begin{array}{l}\text { Increased } \\
\text { Women Own } \\
\text { well-being }\end{array}$ & Yes & Yes & yes & Yes & No \\
\hline $\begin{array}{l}\text { Increased Men } \\
\text { well-being }\end{array}$ & Yes & Yes & no & Yes & No \\
\hline $\begin{array}{l}\text { Poverty } \\
\text { reduction }\end{array}$ & yes & Yes & yes & Yes & $\begin{array}{l}\text { No, even } \\
\text { facing worst } \\
\text { conditions for }\end{array}$ \\
\hline
\end{tabular}




\section{DISCUSSION \& CONCLUSION}

Data shows, that the women who had financial independence available were sharing the responsibilities with men and were getting involved in household decisions. They had power to decide, free to move in the society and have access to market, and were selfdirected, though they are seen succumbed to the cultural influence. Some of them still preferred household related decision be taken by the husbands, fathers, brothers or sons whether or not they are cooperative. Data also showed that in some cases women and men took decisions mutually. On the other hand, the women who transferred their loans to the male family members lacked mobility, social recognition, self-confidence, no improvement in the social status. However, their respect at home was enhanced perhaps because they became the source of credit for their men.

From literature, it was learned that in emerging economies when women had their own businesses, their men withdraw their contribution from their home and eventually women had to bear the family responsibility. However, in this study, there appeared only one case wherein the male head of the family withdrew his contribution from his home. In the other 15 cases, men continuously contributed towards the household with their income. One of the respondent women was widow and lived with her children. But she was independent in her business, financial and household decisions. One woman's husband is out of city and she lived with her parents but independently.

All women were found expending much on the basic necessities like nutrition and health of their children. They were also determined to have them better education. It collectively had improved standard of living of the families that had created a happy atmosphere at their homes. Most of the women narrated in their interview that before securing micro credit, their circumstances were dismal. They said that they would eat only leaving of their children. And occasionally they had to sleep starved. Almost all of them believed that the micro financing had changed their fortune. Their families well realized that their relatives and neighbor give them respect. In one of the cases, however, micro financing could not change fate of the family. The women secured the loan but business was operated by her husband, woman supporting him. But still they were struggling. They could not afford good education to their children.

In this way, our research has supported findings of Sameul et al (2012) that had found out that access micro credit to women has improved their economic conditions, political and social status, and also other factors of life. They say that microfinance interventions had constructed momentous impacts in the families' standard of living; improving their access to medical facilities, education for children, better housing infrastructure, enhancement in employment and income, and creation of employment opportunities for others. Findings of this study also agree with the findings of the study of Mayoux (2005) that concluded that financial stability and women empowerment paradigm were indicating the economic, social and political strength of women, resulting to poverty alleviation as well as improvement in the overall household wellbeing.

This study explored variable of women empowerment qualitatively. This was a difficult task to do since it involves collateral qualitative changes like changes in the controlling process and changes in the relationships. The study concluded that micro financing is a vital tool towards women empowerment in Pakistan. Accordingly, it should be designed holistically and made provided justly to the women from lower strata of the society. 


\section{REFERENCES}

1. Arora, B., \& Singhal, A. (2013), ‘A Comprehensive Literature on Impact of Microfinance', Online International Interdisciplinary Research Journal, III (V), 346-358

2. Basu, J. P. (2006), 'Microfinance and Women Empowerment An Empirical Study with special reference to West Bengal', Indira Ghandi Institute of Development and Research< http://www. igidr. ac. in/money/mfc_10/Jyotish\% 20Prakash\% 20Basu_submission_55.pdf>(cited 2 Mar. 2012), 14.

3. Bhat, R., \& Lakshmi, J. (2002), 'Feminisation of Poverty and Empowerment of Women-An Indian Perspective \& Experience',International Women's Conference, (pp. 6-11). Townsville, Australia.

4. BISP: At a glance. (2007). Retrieved August 18, 2013, from Benazir Income Support Programme: http://www.bisp.gov.pk/

5. CGAP. (2006, April 14). Microfiannce: History of Microfinance. Retrieved September 16, 2013, from Global Envision: exploring market-driven solutions to poverty: http://www.globalenvision.org/library/4/1051

6. Claros, A. L., \& Zahidi, S. (2005), 'Women's Empowerment: Measuring the Global Gender Gap. Geneva, Switzerland', World Economic Forum.

7. Dobra, A., \& Stiftung, F. E. (2011), 'Microfinance: Champion in Poverty Alleviation and Failure in Female empowerment', Munich Personal Repec Archive .

8. Golla, M. A., Malhotra, A., Nanda, P., \& Mehra, R. (2011),'Understanding and Measuring Women's Economic Empowerment',Internation center for research , 69.

9. Hali, S. (2013, May 08). Women Empowerment. Retrieved September 23, 2013, from The Nation: http://www.nation.com.pk/pakistan-news-newspaper-dailyenglish-online/columns/08-May-2013/women-empowerment

10. Hans, V. B. (2009), 'Innovations in Microfinance: Looking beyond income poverty', Found on Microfinance Gateway Website: http://www. Microfinance gateway. org/gm/document-1.9, 35223 .

11. Hunt, J., \&Kasynathan, N. (2002), 'Reflections on microfinance and women's empowerment', Development Bulletin, 57, 71-75.

12. Ifelunini, I. A., \& Wasowei, E. C. (2013), 'Constraints to Women Entrepreneurs' Access to Microfinance in South-South Nigeria', Research journal of Finance and Accounting, 4 (6).

13. Jamal, H. (2008), 'Exploring the impact of Microfinance in Pakistan' Social Policy and Development Centre.

14. Kabeer, N. (2002), 'Conceptualizing empowerment as a transformative strategy for Poverty Eradication and the Implication for the Measuring Progress',Empowerment of women throught the life cycle as a tranformative strategy for poverty eradication.

15. Lemire, B., Pearson, R., \& Campbell, G. G. (2001),'Women and Credit: Researching the Past, Refiguring the Future',(Vol. 24). Berg.

16. Luttrell, C., Quiroz, S., Scrutton, C., \& Bird, K. (2009), 'Understanding and operationalising empowerment', London, UK: Overseas Development Institute.

17. Mayoux, L. (2005),'Women empowerment through sustainable microfinance, Pakistan',Aga Khan foundation Canada.

18. Mayoux, L. C. (2006), 'Women's empowerment through sustainable microfinance: Rethinking 'best practice'”.

19. Meiring, M. (1996), 'Women Involvement: Empowerment or Disempowerment',Reaching The Unreached: Challenges For The 21st Century(pp. 139-140). New Delhi, India: Water, Engineering and Development Center.

20. Montgomery, H., \& Weiss, J. (2011), 'Can commercially-oriented microfinance help meet the millennium development goals? Evidence from Pakistan', World Development, 39(1), 87-109.

21. Nasir, S. (2013), 'Microfinance in India: Contemporary Issues and Challenges', Middle-East Journal of Scientific Research, 15(2), 191-199.

22. Nair, S. (2013), 'The rise of womenhood.org',. Social Empowerment of women .

23. Otero, M. (1999), 'Bringing development back, into microfinance', Journal of Microfinance/ESR Review, 1(1), 8-19. 
24. Ray, W. (1994), 'Reading women: cultural authority, gender, and the novel: The case of Rousseau', Eighteenth-century studies, 27(3), 421-447.

25. Sutton-Brown, C. (2011), 'Women's Empowerment in the Context of Microfinance: A Photovoice Study'.

26. Sameul, A., Sharon, A.-A., Charlotte, B., Ekow, C. F., \& Mary, K. (2012), 'Empowerment of Rural Women: The Role of Microfinance Institutions', Christian Service University College.

27. Sekaran, U.\&Bougie, R. (2010), 'Research Methods for Business: A Skill Building Approach', UK: John Wiley \& Sons

28. Schreiner, M., \&Colombet, H. H. (2001), 'From urban to rural: Lessons for microfinance from Argentina', Development Policy Review, 19(3), 339-354.

29. Thibos, M., Lavin-Loucks, D., \& Martin, M. (2007), 'The Feminization of Poverty. Dallas TX',The J. McDonald Williams Institute \& YWCA. Retrieved December,7, 2007.

30. Topouzis, D. (1990),'Feminization of Poverty’, Africa Report, 35(3), 60-63.

31. Sinclair, T. J. (2012), 'Beyond the Control: the Disempowerment of Women in Middle Eastern and African Literature',East Carolina University.

32. Ul, H. A. (2013), 'Management of microfinance Programmes and their contribution to poverty alleviation'.

33. UNCDF and development themes: Women's and Youth Empowerment. (n.d.), Retrieved August 18, 2013, from United Nations Capital Development Funds: http://www.uncdf.org/en/womens-empowerment

34. UNDP: Women's Empowerment. (n.d), Retrieved August 19, 2013, from United Nations Development Programme: http://www.undp.org/content/undp/en/home/ourwork/womenempowerment/overvie w.html 